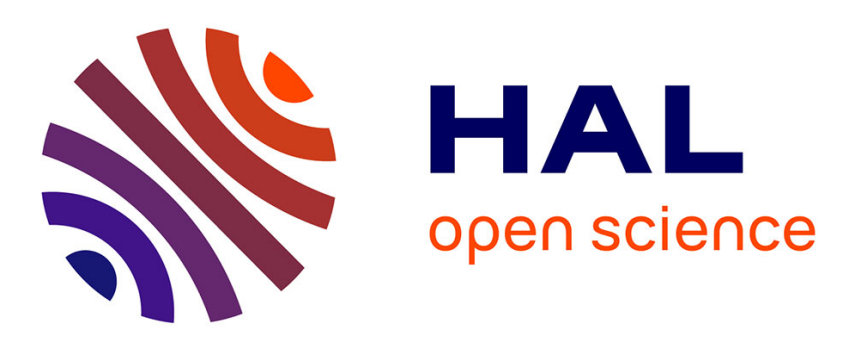

\title{
Effet de la température sur l'expression des symptômes de la prolifération du pommier
}

Jean-Pierre Ducroquet, Françoise Dosba, Micheline Lansac, Kléber Mazy

\section{To cite this version:}

Jean-Pierre Ducroquet, Françoise Dosba, Micheline Lansac, Kléber Mazy. Effet de la température sur l'expression des symptômes de la prolifération du pommier. Agronomie, 1986, 6 (10), pp.897-903. hal-00884837

\section{HAL Id: hal-00884837 https://hal.science/hal-00884837}

Submitted on 1 Jan 1986

HAL is a multi-disciplinary open access archive for the deposit and dissemination of scientific research documents, whether they are published or not. The documents may come from teaching and research institutions in France or abroad, or from public or private research centers.
L'archive ouverte pluridisciplinaire HAL, est destinée au dépôt et à la diffusion de documents scientifiques de niveau recherche, publiés ou non, émanant des établissements d'enseignement et de recherche français ou étrangers, des laboratoires publics ou privés. 


\title{
Effet de la température sur l'expression des symptômes de la prolifération du pommier
}

\author{
Jean-Pierre DUCROQUET, Françoise DOSBA $\left({ }^{*}\right)$, Micheline LANSAC $\left({ }^{*}\right) \&$ Kléber MAZY $\left({ }^{*}\right)$ \\ Estaçao Experimental, EMPASC 89560, Videira SC, Brésil. \\ (*) I.N.R.A., Station de Recherches d'Arboriculture Fruitière, Centre de Recherches de Bordeaux, B.P. 31 , \\ La Grande Ferrade, F 33140 Pont-de-la-Maye.
}

Mots clés additionnels : $M L O$, Catharanthus roseus G. Don, DAPI.

\section{INTRODUCTION}

Depuis que la prolifération du pommier a été décrite en 1950 par Rui dans le Nord de l'Italie, cette maladie a été signalée dans tous les pays d'Europe mais pas encore en Amérique.

Après la découverte des organismes de type mycoplasme (MLO) dans les tubes criblés de plantes atteintes de jaunisses et autres maladies de dégénérescence (Dol et al., 1967), différents travaux ont montré que la prolifération du pommier était associée à la présence de MLO dans les tubes criblés des arbres atteints (GIANNOTI et al., 1968; MARWITZ et al., 1974).

L'utilisation du fluorochrome spécifique de l'ADN, le $4^{\prime}-6$, diamidino 2-phénylindole ou DAPI, a permis de confirmer, sur un nombre important d'échantillons, les résultats d'examens en microscopie électronique (SCHAPER \& SEemuller, 1982 ; DuCroquet, 1985).
Les MLO sont dépourvus de paroi et de ce fait sont insensibles à la pénicilline qui normalement agit sur la synthèse du peptidoglycane, mais leur multiplication est inhibée par des antibiotiques comme l'oxytétracycline (ISHIE et al., 1967 ; COUSIN \& STARON, 1969 ; Bove, 1984). Chez de jeunes pommiers malades cultivés en pots, la pénicilline à $1 \mathrm{~g} / \mathrm{l}$ appliquée par immersion du système racinaire s'avère totalement inefficace alors que l'oxytétracycline à $0,5 \mathrm{~g} / \mathrm{l}$ appliquée dans les mêmes conditions aboutit à une rémission complète des symptômes et à la disparition des microorganismes associés à la prolifération, tout au moins dans la partie aérienne (DUCROQUET, 1985) ; ceci confirme que ces micro-organismes sont démunis de paroi et renforce l'hypothèse selon laquelle les agents pathogènes de la prolifération du pommier seraient des MLO.

Néanmoins, si certains MLO ont pu être isolés (SINHA, 1979 ; MEIGNOZ et al., 1982) aucun n'a été 
cultivé jusqu'à maintenant; la démonstration de pathogénie selon le postulat de KOCH n'a pu être faite et la connaissance de ces organismes a encore peu progressé.

Les exigences thermiques d'un organisme constituent un élément de caractérisation dont la connaissance permet non seulement de le différencier d'un autre organisme, mais encore de préciser les conditions d'expérimentation relatives à cet organisme.

KUNKEL (1937) avait montré que des cicadelles maintenues pendant 12 jours ou plus à une température de $32{ }^{\circ} \mathrm{C}$, étaient devenues incapables de transmettre la maladie de l'aster yellow, alors que, pour une durée inférieure, l'incapacité n'était que temporaire.

Bove et al. (1974) ont mis en évidence l'effet de la température sur l'expression des symptômes chez des agrumes atteints de différentes maladies associées à la présence de procaryotes dans les tubes criblés. NAKAMURA et al. (1978) ont obtenu des résultats similaires dans le cas de la phyllodie du trèfle.

Afin de déterminer l'effet de la température sur l'expression des symptômes et les populations de MLO, nous avons conduit plusieurs essais en chambres climatisées et en serres avec des pervenches de Madagascar (Catharanthus roseus G. DON) et des pommiers atteints de prolifération.

\section{MATÉRIELS ET MÉTHODES}

\section{A. Matériel végétal}

Il est constitué de pervenches de Madagascar issues de semis et de jeunes plants de pommier obtenus par bourgeonnement de racines.

Les pervenches ont été inoculées 50 jours après le semis par greffage en fente de pointes apicales prélevées sur des pervenches contaminées par la souche « Marwitz » de prolifération du pommier (MARWITZ et al., 1974). Cette souche a été isolée après transmission par pont cuscute entre pommier atteint de prolifération et une pervenche de Madagascar. Elle a été multipliée par greffage herbacé de pervenche malade à pervenche saine (fig. 1a). Les symptômes observés sont : une diminution de la taille et du nombre de fleurs, une diminution de la taille des feuilles avec déformation dissymétrique du limbe qui présente un jaunissement marginal et un éclaircissement des nervures (fig. 1b).

La technique du bourgeonnement de racines (RoBINSON \& SCHWABE, 1977) nous a permis d'obtenir, à partir de pommiers sains ou atteints de prolifération, des jeunes plants sains ou contaminés en totalité par cette maladie.

Ces racines provenaient de deux porte-greffes différents : MI 793 prélevé dans un verger atteint de différentes maladies à virus, en particulier de Chlorotic leaf spot virus (CLSV) et de Spy decline, mais sans bois caoutchouc, et MM 106 prélevé dans un verger indemne de maladie à virus à la plantation et sans CLSV au moment de l'expérimentation. Les arbres sur lesquels ont été effectués les prélèvements présentaient des symptômes typiques de prolifération depuis plusieurs années.

\section{B. Conditions expérimentales}

Nous disposions de 2 chambres climatisées et de 2 serres:

Les chambres climatisées étaient soumises à une photopériode de 6 heures d'obscurité et 18 heures d'éclairage artificiel à raison de 40 à 60 microeinsteins $\mathrm{m}^{-2} \mathrm{~s}^{-1}$ au niveau des plantes, selon leur hauteur :

- chambre tempérée: température de $21^{\circ} \mathrm{C}$ à l'obscurité ou $24^{\circ} \mathrm{C}$ à la lumière, et hygrométrie de 80 à 90 p. 100 ;

- chambre chaude : température de $29^{\circ} \mathrm{C}$ à l'obscurité ou $32^{\circ} \mathrm{C}$ à la lumière, et hygrométrie de 50 à 70 p. 100.

Les serres utilisées bénéficiaient d'un certain contrôle de température et d'humidité avec un éclairage d'appoint pour compléter la durée du jour lorsque celle-ci était inférieure à $16 \mathrm{~h}$. Cependant les oscillations journalières étaient importantes et la différence de température entre les deux serres était assez faible :

- serre tempérée : $22{ }^{\circ} \mathrm{C}$ la nuit et $30^{\circ} \mathrm{C}$ le jour

- serre chaude : $24^{\circ} \mathrm{C}$ la nuit et $34{ }^{\circ} \mathrm{C}$ le jour.

\section{Méthodologie}

Un premier essai a été conduit avec des pervenches de Madagascar en chambres climatisées.

Quarante et une pervenches atteintes de prolifération (AP) (tabl. 3) et 24 pervenches saines autogreffées ont été réparties entre les 2 chambres, en proportions égales, 13 jours après le greffage d'inoculation. Cent jours après ce greffage la moitié des plants AP de chaque chambre était transférée dans l'autre chambre.

Un second essai a été conduit avec 56 pommiers MM 106 (AP) et $63 \mathrm{MM} 106$ sains qui ont été répartis entre chambre tempérée $\left(21-24^{\circ} \mathrm{C}\right)$, chambre chaude $\left(29-32^{\circ} \mathrm{C}\right)$, serre tempérée $\left(22^{\circ}-30^{\circ}\right)$ et serre chaude $\left(24^{\circ}-34^{\circ} \mathrm{C}\right)$. Cette répartition a été effectuée lorsque les plantes AP mesuraient en moyenne $15 \mathrm{~cm}$ de hauteur et les plants sains $30 \mathrm{~cm}$, bien qu'ils aient été bouturés en même temps.

Pour ces deux essais, nous avons fait des mesures hebdomadaires de croissance et des observations périodiques de l'évolution des symptômes. Pour les pervenches, on a mesuré la croissance du greffon et de l'axe secondaire provenant de l'un des 2 bourgeons situés immédiatement en dessous du point de greffage, l'autre ayant été supprimé 2 semaines après le greffage (fig. 1a). La détection des MLO dans des tubes criblés a été réalisée par le test DAPI (SEEMULLER, 1976 ; DOSBA \& LANSAC, 1982).

\section{RÉSULTATS}

\section{A. Effet de deux niveaux de température sur la per- venche de Madagascar atteinte de prolifération}

L'évolution des symptômes en fonction des températures (tabl. 1) montre que des températures élevées 
TABLEAU

Evolution comparée des symptômes et des réponses au test DAPI chez les pervenches saines et malades en fonction de la température. Compared development symptom and DAPI responses in healthy and diseased (AP) periwinkles according to the temperature conditions.

\begin{tabular}{|c|c|c|}
\hline $\begin{array}{l}\text { Nbre de jours } \\
\text { après le greffage }\end{array}$ & Chambre chaude $\left(29-32^{\circ} \mathrm{C}\right)$ & Chambre tempérée $\left(21-24^{\circ} \mathrm{C}\right)$ \\
\hline 38 & Développement similaire des greffons sains et AP. & $\begin{array}{l}\text { Légère croissance des greffons sains, état stationnaire } \\
\text { des greffons AP. }\end{array}$ \\
\hline 42 & Aucune différence entre plants sains et plants AP. & $\begin{array}{l}\text { Symptômes typiques de prolifération sur plants inoculés } \\
\text { (jaunisse et ondulation de la bordure des feuilles). Plants } \\
\text { sains sans symptômes. }\end{array}$ \\
\hline 66 & Croissance soutenue des pervenches saines et AP. & Croissance faible surtout chez les pervenches AP. \\
\hline 75 & $\begin{array}{l}\text { Réponse négative au test DAPJ sur les pétioles de toutes } \\
\text { les pervenches inoculées, sauf le } n^{\circ} 35 \text { qui présente un } \\
\text { jaunissement. Floraison normale sur les plants inoculés et } \\
\text { sains. }\end{array}$ & $\begin{array}{l}\text { Réponse positive au test DAPI sur les pétioles de toutes les } \\
\text { pervenches inoculées. Floraison normale seulement chez les } \\
\text { plantes témoins auto-greffées. }\end{array}$ \\
\hline 100 & $\begin{array}{l}\text { Passage de la moitié des plantes en chambre tempérée: } \\
\text { le } n^{\circ} 35 \text { est resté en chambre chaude. }\end{array}$ & Passage de la moitié des plantes en chambre chaude. \\
\hline 115 & $\begin{array}{l}\text { Régression des symptômes chez les plants venant de } \\
\text { chambre tempérée. }\end{array}$ & $\begin{array}{l}\text { Parmi les plants venant de chambre chaude, une pervenche } \\
\text { présente des symptomes de prolifération. }\end{array}$ \\
\hline 148 & $\begin{array}{l}\text { Rémission complète les symptômes de prolifération, néan- } \\
\text { moins } 3 \text { plantes resces en chambre chaude (dont le n }{ }^{\circ} 35 \text { ) } \\
\text { présentent une jaunisse associée à la présence de MLO. }\end{array}$ & $\begin{array}{l}\text { Symptômes accentués chez les plants toujours maintenus en } \\
\text { chambre tempérée. } 2 \text { parmi ceux provenant de chambre } \\
\text { chaude présentent des symptômes. }\end{array}$ \\
\hline
\end{tabular}

(a)

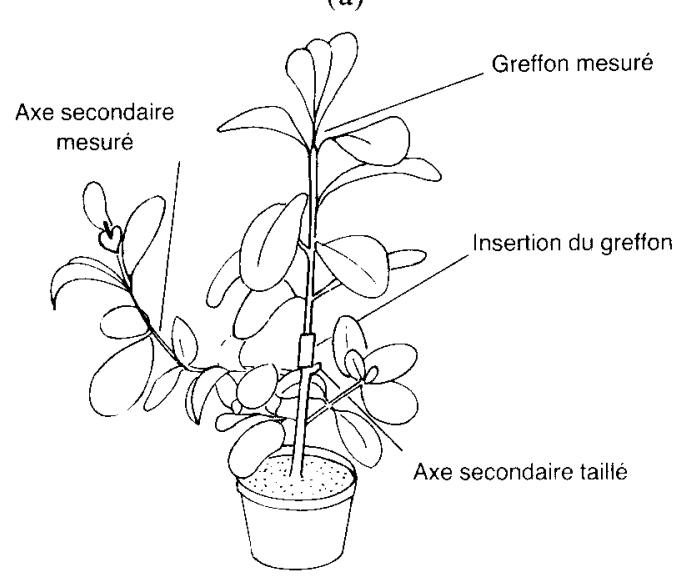

(b)

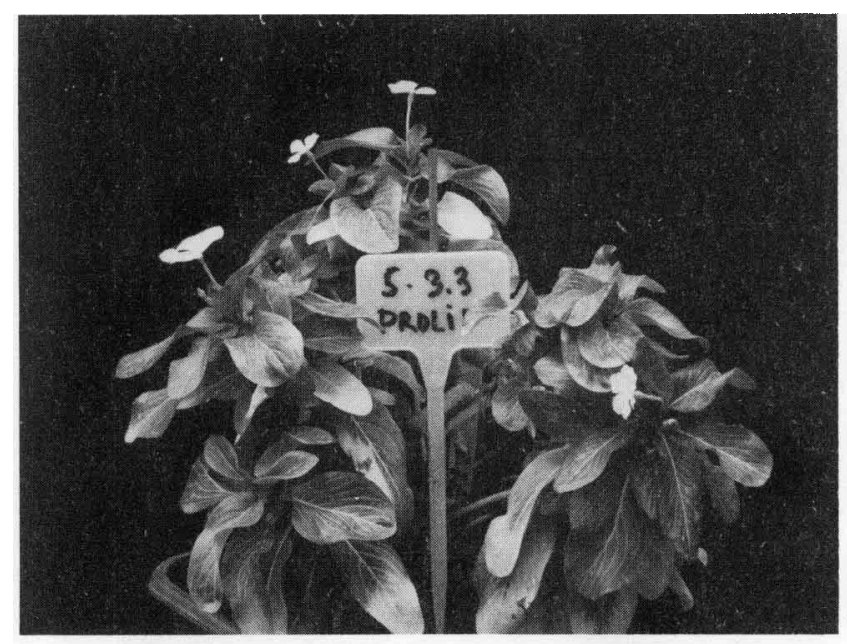

Figure 1

a) Plante greffée de pervenche

b) Sympiômes sur pervenche après inoculation de la prolifération du pommier.

a) Grafted plant of periwinkle.

b) Symptoms on periwinkle after apple proliferation graft inoculation.
(29-32 ${ }^{\circ} \mathrm{C}$ ) inhibent nettement l'expression des symptômes de prolifération alors que ceux-ci sont très nets chez les pervenches soumises aux températures plus basses $\left(21-24^{\circ} \mathrm{C}\right)$.

On n'a pas retrouvé de MLO par le test DAPI dans les pétioles de pervenches AP de la chambre chaude sauf chez une plante dont les symptômes de jaunisse n'étaient pas accompagnés des symptômes typiques de la prolifération du pommier notamment l'éclaircissement des nervures et la déformation du limbe (tabl. 1).

La croissance des pervenches, inoculées ou non, a été nettement plus faible en chambre tempérée qu'en chambre chaude ; la différence entre plants sains et plants inoculés a été très marquée en chambre tempérée, surtout au niveau du greffon (tabl. 2).

\section{TABLEAU 2}

Hauteur (en $\mathrm{cm}$ ) de pervenches, 66 jours après le greffage d'inoculation, selon l'état sanitaire des plants et la température. Height (in $\mathrm{cm}$ ) of periwinkles, 66 days after inoculation, according to the sanitary and the temperature condition.

\begin{tabular}{llc}
\hline \hline & $\begin{array}{c}\text { Chambre } \\
\text { chaude }\end{array}$ & $\begin{array}{c}\text { Chambre } \\
\text { tempérée }\end{array}$ \\
\hline Greffon sain & $27,6 \mathrm{a}\left({ }^{*}\right)$ & $9,6 \mathrm{~b}$ \\
Greffon malade & $21,9 \mathrm{a}$ & $4,2 \mathrm{a}$ \\
Axe secondaire sain & $23,0 \mathrm{a}$ & $10,4 \mathrm{~b}$ \\
Axe secondaire malade & $20,8 \mathrm{a}$ & $7,4 \mathrm{a}$ \\
\hline \hline
\end{tabular}

(*) Deux lettres différentes expriment une différence significative entre les moyennes correspondantes au seuil $5 \%$.

La hauteur des pervenches en chambre chaude ne diffère pas significativement mais, en chambre tempérée, les plants sains sont significativement plus grands que les plants malades (tests de Duncan au seuil 5 p. $100)$. 
Les observations faites après le transfert de la moitié des pervenches d'une chambre à l'autre, confirment l'effet de la température sur l'expression des symptômes et la progression des MLO (tabl. 3).

TABLEAU 3

Evolution des symptômes et des réponses au test DAPI chez les pervenches inoculées, selon le régime de températures et le nombre de jours après l'inoculation.

Symptom development and DAPI responses in infected periwinkles according to the temperature and the number of days after inoculation.

\begin{tabular}{|c|c|c|c|c|}
\hline \multirow{2}{*}{$\begin{array}{c}\text { Régime } \\
\text { de } \\
\text { température }\end{array}$} & \multicolumn{2}{|c|}{ Symptômes $(+)$} & \multicolumn{2}{|c|}{ DAPI $(+)$} \\
\hline & $100 \mathrm{j}$. & $148 \mathrm{j}$. & $\begin{array}{c}\text { sur } \\
\text { pétioles } \\
148 \mathrm{j} \text {. }\end{array}$ & $\begin{array}{c}\text { sur } \\
\text { racines } \\
170 \mathrm{j} \text {. }\end{array}$ \\
\hline Chambre chaude & $1 / 9\left(^{*}\right)$ & $3 / 9\left(^{*}\right)$ & $3 / 9\left(^{*}\right)$ & $3 / 9(*)$ \\
\hline $\begin{array}{l}\text { Chambre tempérée après } \\
\text { chambre chaude }\end{array}$ & $0 / 12$ & $2 / 12$ & $2 / 12$ & - \\
\hline $\begin{array}{l}\text { Chambre tempérée } \\
\text { Chambre chatude apres }\end{array}$ & $10 / 10$ & $10 / 10$ & - & - \\
\hline chambre tempérèe & $10 / 10$ & $0 / 10$ & $1 / 10$ & $10 / 10$ \\
\hline
\end{tabular}

(*) Nombre de pervenches symptomes $(+)$ ou DAPI $(+) /$ nombre total de pervenches.

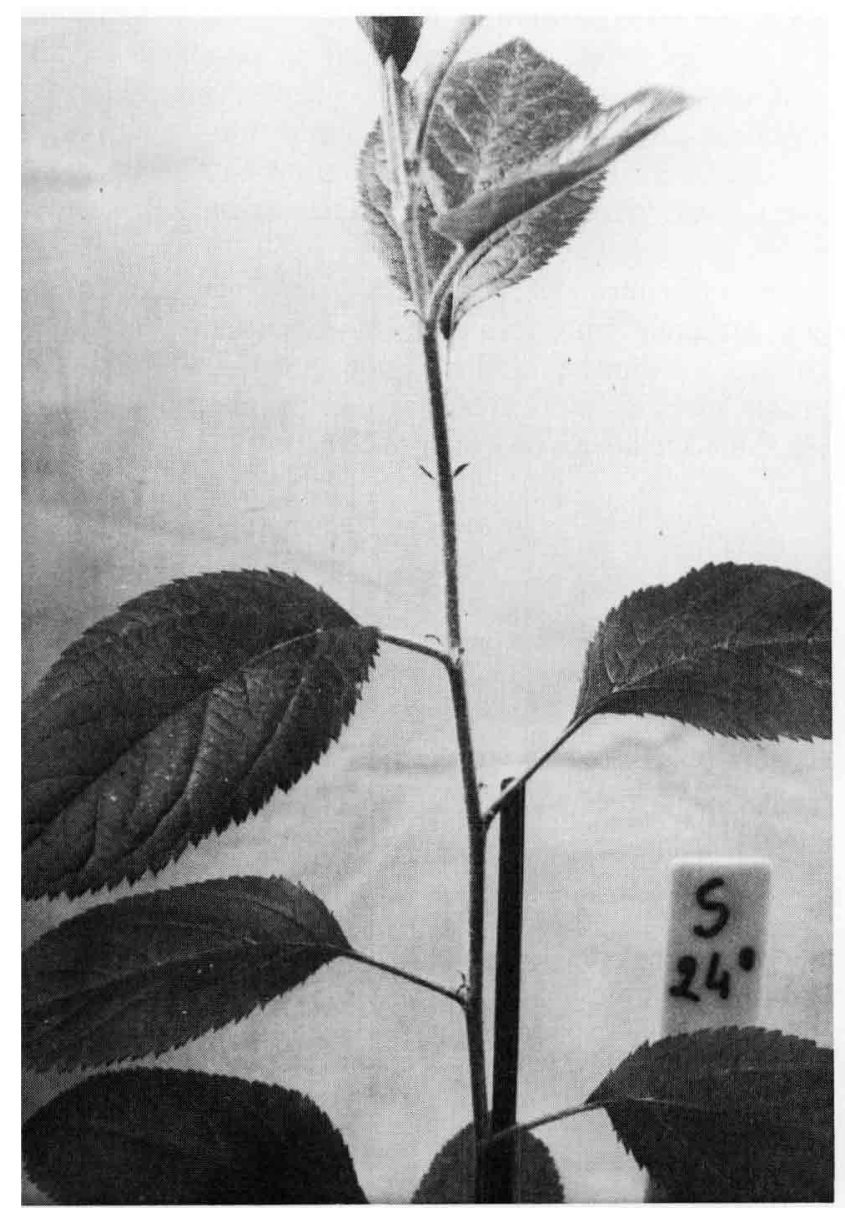

Figure 2

Chambre tempérée (21-24 $\left.{ }^{\circ} \mathrm{C}\right)$.

a) Pommier MI 793, sain, partie supérieure.

b) Pommier MI 793 AP. Partie supérieure avec départs anticipés de bourgeons axillaires.
En effet les symptômes s'accentuent chez les plants (AP) maintenus en chambre tempérée et disparaissent chez les plants passés en chambre chaude après séjour en chambre tempérée, bien que, dans ce cas, les MLO, se soient maintenus dans les racines.

Parmi les 12 plants passés en chambre tempérée à partir de la chambre chaude, 2 ont présenté des symptômes, montrant ainsi que, pour au moins une partie des plants maintenus pendant 2 mois en chambre chaude, les MLO n'avaient pas disparu totalement.

Parmi les 9 pervenches maintenues en chambre chaude, 3 plants dont le $\mathrm{n}^{\circ} 35$ ont présenté des symptômes de jaunisse sans exprimer les symptômes typiques de prolifération. Nous n'avons pu déterminer la cause de cette anomalie, cependant nous avons vérifié que ces symptômes, différents de ceux de la prolifération du pommier, étaient néanmoins associés à la présence de MLO dans les tubes criblés.

\section{B. Effet du facteur température sur l'expression des symptômes de prolifération chez le pommier}

L'essai conduit sur MM 106 simultanément en serres et en chambres climatisées a confirmé les résultats obtenus avec la pervenche (tabl. 4).

En chambre chaude, aucun pommier n'a présenté les symptômes de balais de sorcières caractéris-

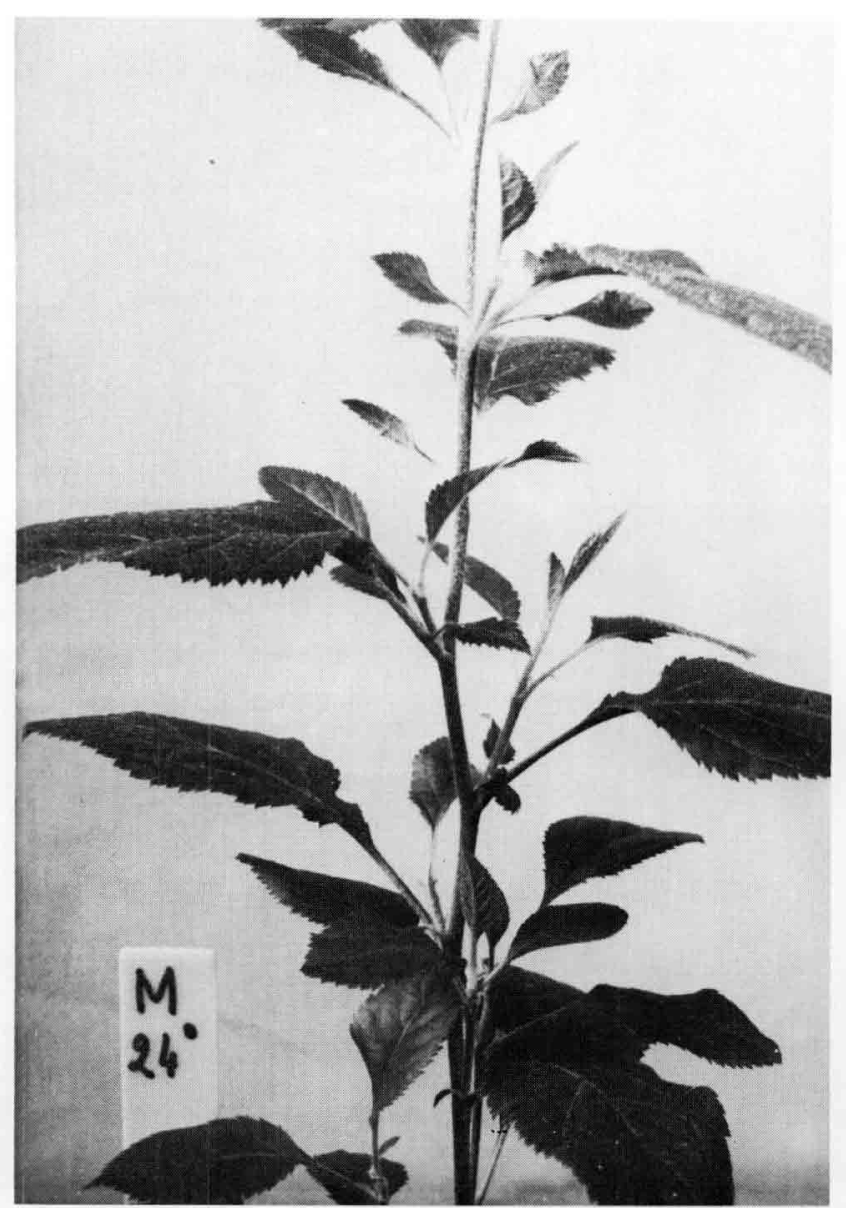

Temperate room $\left(21-24^{\circ} \mathrm{C}\right)$.

a) Healthy apple plant MI 793. Upper part.

b) Apple plant MI 793 AP. Upper part with early axillary bud burst. 
TABLEAU 4

Proportion de pommiers MM 106 présentant des " balais de sorcière " selon les conditions de milieu. Proportion of MM 106 apple rootstocks showing 'witches broom' in different climatic conditions.

\begin{tabular}{lcc}
\hline \multicolumn{1}{c}{ Environnement } & Plants malades & Plants sains \\
\hline Chambre chaude & $0 / 15$ & $0 / 9$ \\
Chambre tempérée & $11 / 13$ & $0 / 11$ \\
Serre chaude & $10 / 14$ & $0 / 20$ \\
Serre tempérée & $10 / 14$ & $0 / 23$ \\
\hline \hline
\end{tabular}

tiques de la prolifération. Au contraire, en chambre tempérée, les symptômes se sont exprimés de façon très nette chez la majorité des pommiers infectés.

Dans les serres, les températures diurnes élevées n'ont pas empêché l'expression des symptômes qui se sont présentés de la même façon que dans la chambre tempérée. On peut donc supposer que l'effet des températures diurnes élevées a été interrompu par des températures nocturnes trop basses et a donc été peu marqué.
Les tests DAPI effectués en fin d'essai sur pommiers maintenus en chambre chaude confirment l'élimination des MLO de la partie aérienne, tandis que leur présence a pu être détectée dans le système racinaire de 14 et 15 plants testés.

Ces résultats confirment ceux obtenus lors d'essais préliminaires conduits avec le pommier MI 793 dans les mêmes conditions, et dont les symptômes en chambre tempérée sont présentés à la figure 2. Etant donné le mauvais état sanitaire des porte-greffes MI 793, on peut penser que la présence simultanée de virus et de MLO n'altère en rien la sensibilité des MLO à l'action de la température.

L'établissement des courbes de croissance des plants MM 106 sains ou malades en chambres climatisées a montré une évolution différente selon la température. Dans la chambre tempérée l'écart entre les courbes de croissance des plants sains et malades est important (fig. 3).

En chambre chaude, la courbe de croissance des plants malades s'est rapprochée progressivement de celle des plants sains de telle sorte que leurs intervalles de confiance se sont chevauchés (fig. 4).

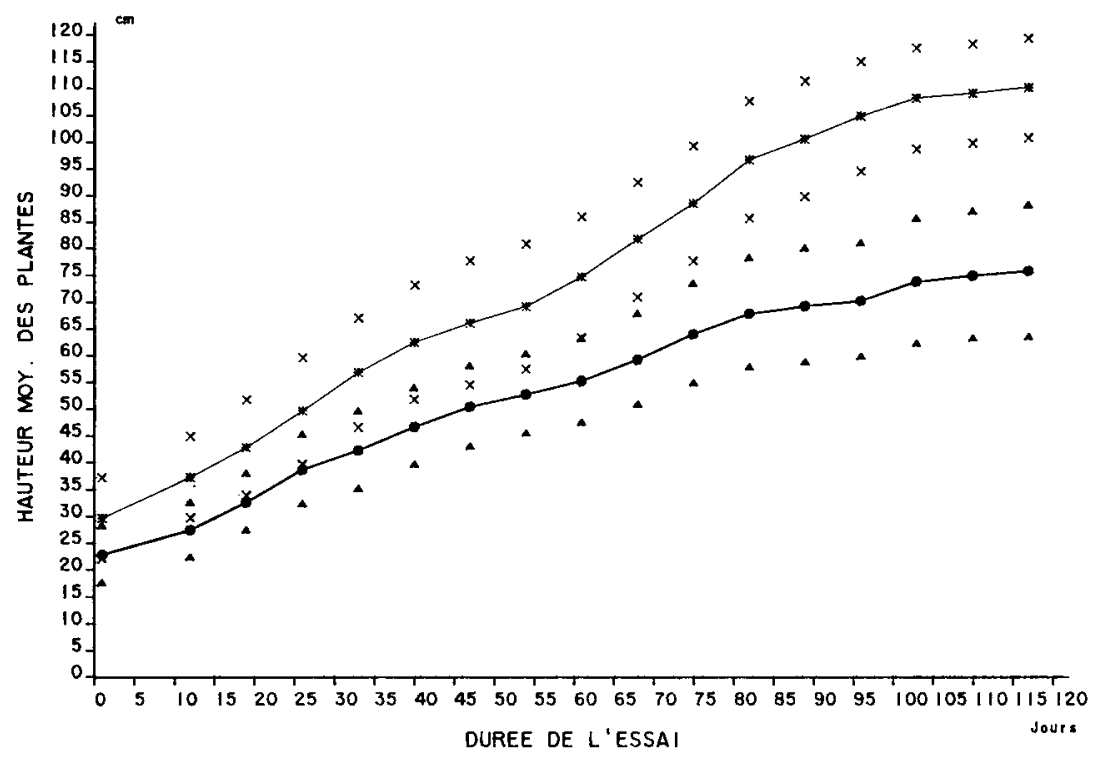

Figure 3

Croissance comparée des pommiers MM 106 sains et malades en chambre tempérée $\left(21-24^{\circ} \mathrm{C}\right)$.

- Moyenne plantes malades.

- Limites supérieure et inférieure de l'intervalle de confiance des plantes malades.

* Moyenne plantes saines.

$\times$ Limites supérieure et inférieure de l'intervalle de confiance des plantes saines.

Comparison of the growth of healthy and diseased apple plants $M M 106$ in a temperate room $\left(21-24^{\circ} \mathrm{C}\right)$.

\section{Figure 4}

Croissance comparée des pommiers MM 106 sains et malades en chambre chaude $\left(29-32^{\circ} \mathrm{C}\right)$.

- Moyenne plantes malades.

- Limites supérieure et inférieure de l'intervalle de confiance des plantes malades.

* Movenne plantes saines.

$\times$ Limites supérieure et inférieure de l'intervalle de confiance des plantes saines.

Comparison of the growth of healthy and diseased apple MM 106 in a hot room $\left(29-32^{\circ} \mathrm{C}\right)$.

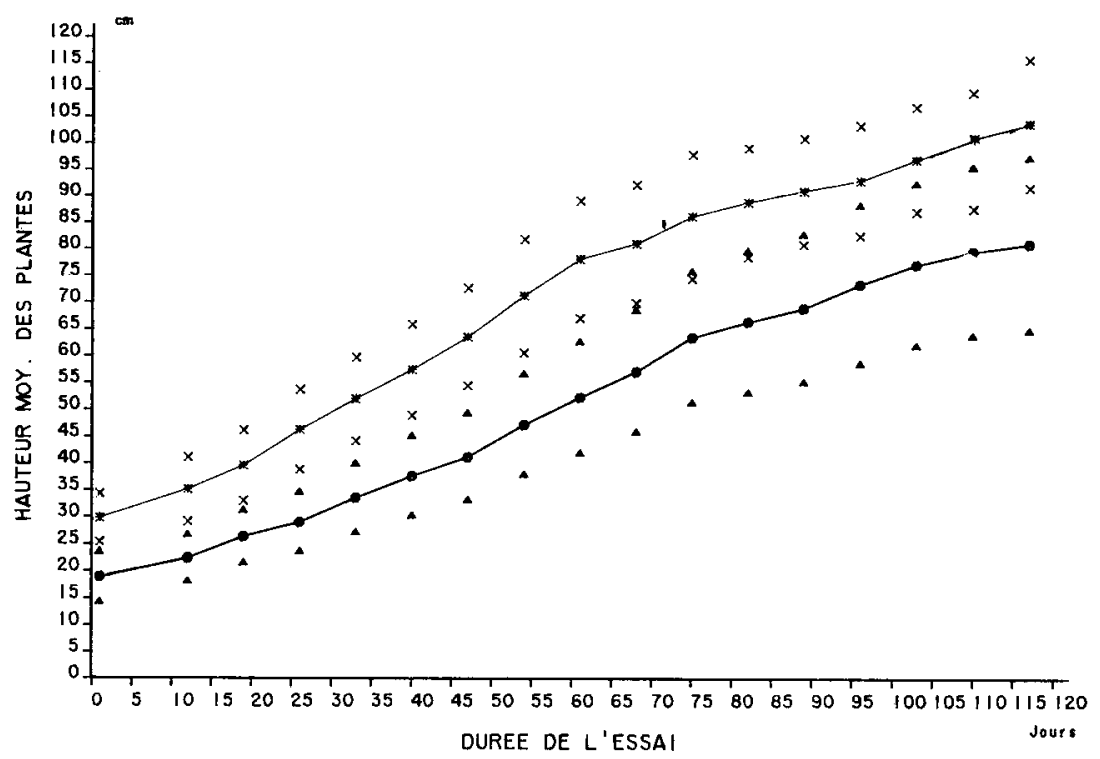


Dans les serres chaudes et tempérées, les écarts entre les courbes de croissance ont été identiques et du même ordre que celui observé en chambre tempérée bien que la croissance ait été un peu plus faible.

\section{DISCUSSION - CONCLUSION}

La similitude des effets provoqués par la température chez des hôtes aussi différents que la pervenche de Madagascar, une plante herbacée de climat tropical, et le pommier, une plante ligneuse de climat tempéré, semble bien indiquer qu'il s'agit d'une sensibilité spécifique des MLO associés à la prolifération du pommier et non d'une réaction physiologique de la plante à ces MLO en fonction de la température. On pourrait en avoir une confirmation en maintenant aussi le système racinaire des plantes atteintes, aux températures situées entre 29 et $32^{\circ} \mathrm{C}$. En effet, la permanence des MLO dans les racines des plants maintenus en chambre chaude peut être attribuée à une température plus basse de $4{ }^{\circ} \mathrm{C}$ dans les pots par rapport à la température de la chambre, mesurée à la fin de l'essai.

Les températures situées entre 21 et $24{ }^{\circ} \mathrm{C}$ sont favorables à l'expression des symptômes et à la multiplication des MLO associés à la prolifération mais ne constituent peut-être pas un optimum. Celui-ci pourrait être déterminé de façon plus précise.

L'expression de symptômes de jaunisse chez 3 pervenches maintenues en chambre chaude résulte peut-être d'une contamination accidentelle par une souche de MLO différente de celle associée à la prolifération du pommier et résistante à des températures allant jusqu'à $32^{\circ} \mathrm{C}$. Ces symptômes ont été reproduits chez des pervenches inoculées à partir des 3 pervenches présentant des symptômes atypiques, et maintenues en chambre chaude ou tempérée. On peut aussi envisager pour expliquer ces symptômes qu'une sélection se soit opérée dans la population des MLO sous l'effet de la température. Seuls les MLO se multipliant à température élevée auraient colonisé la plante qui, à partir d'une certain seuil de multiplication, extérioriserait des symptômes.
Un comportement similaire à celui de ces 3 pervenches a ensuite été observé chez des pervenches soumises aux mêmes conditions de température et auxquelles on avait transmis des maladies associées à des MLO, affectant le genre Prunus ainsi qu'une maladie de même type affectant une pervenche contaminée naturellement dans les environs de Bordeaux (Ile d'Arsins, 1976).

La disparition des symptômes de prolifération du pommier chez des plantes maintenues à des températures situées entre $29^{\circ} \mathrm{C}$ et $32^{\circ} \mathrm{C}$ n'explique pas l'absence de cette maladie dans des pays comme le Brésil, puisque dans les régions où sont implantés la majorité des vergers de pommiers de ce pays, la moyenne des températures minimales du mois le plus chaud dépasse rarement $17^{\circ} \mathrm{C}$ et la moyenne des températures maximales est de l'ordre de $28{ }^{\circ} \mathrm{C}$, soit des températures au-dessous de celles qui ont permis l'élimination des MLO dans les conditions expérimentales de ce travail. Il est par ailleurs probable que la maladie ait été introduite dans ce pays puisque la plupart des pieds mères ont été importés d'Europe entre 1965 et 1975, lorsque les techniques d'indexation présentaient des résultats très aléatoires pour la détection de la prolifération du pommier (SCHMID, 1965). Comme les écarts de température diurne-nocturne observés au Brésil sont analogues à ceux observés dans nos expériences conduites en serre, on peut penser qu'à long terme des températures élevées même avec de fortes oscillations journalières soient néfastes aux MLO et à l'expansion de la prolifération dans ce pays. L'interaction température-durée reste à préciser.

La mise en évidence de températures favorables (21$24^{\circ} \mathrm{C}$ ) à l'expression des symptômes de prolifération et donc à la multiplication des MLO, pourra contribuer à l'obtention de meilleurs résultats pour toute expérimentation relative à la prolifération du pommier et en particulier pour les essais de transmission et de mise en culture in vitro.

Reçu le 17 décembre 1985 Accepté le 9 septembre 1986.

RÉFÉRENCES BIBLIOGRAPHIQUES

Bove J. M., 1984. Wall-less prokaryotes of plants. Ann. Rev. Phytopathol., 22, 361-396.

Bove J. M., Calavan E. C., Capoor S. P., Cortez R. E., Schwarz R. E., 1974. Influence of temperature on symptoms of California stubborn, South Africa greening, India Citrus decline, and Philippines leaf mottling disease. In Proceeding of the sixth conference of international organisation of citrus virologists. 1-11. 11., Ed. L. G. Weathers and M. Cohen, University of California Press.

Cousin M. T., Staron T., 1969. Action de quelques antibiotiques sur les maladies végétales causées par des micro-organismes apparentés au groupe des mycoplasmes ou de PLT. Ann. Phytopathol., $1,267-274$

Doi Y., Teranaka M., Yoka K., Asuyama H., 1967. Mycoplasma or PLT group like micro-organism found in the phloem elements of plants infected with mulberry dwarf, potato witches' broom, aster yellow or paulownia witches' broom. Ann. Phytopath. Soc., Japan, 33, 259-266.
Dosba F., Lansac M., 1982. Detection des organismes de type mycoplasme chez les espèces fruitières au moyen du test DAPI. $2^{e}$ Colloque sur les recherches fruitières Bordeaux, 247-257.

Ducroquet J. P., 1985. Maladie de la prolifération du Pommier. Influence de différents facteurs sur les populations de MLO et l'expression des symptômes. Thèse de Docteur Ingénieur, Biologie et Physiologie Végétale. Université de Bordeaux II, $\mathrm{n}^{\circ} 23$.

Giannoti J., Morvan G., Vago C., 1968. Micro-organismes de type mycoplasme dans les cellules libériennes de Malus sylvestris L. atteint de la maladie des proliférations. C. R. Acad. Sc., Paris, série $\mathrm{D}, 267,76-77$

Ishiie T., Doi Y., Yora K., Asuyama H., 1967. Suppressive effects of antibiotics of tetracycline group on symptom development of mulberry dwarf disease. Ann. Phytopath. Soc., Japan, 33, 267-275.

Kunkel L. O., 1937. Effect of heat on ability of Cicadula sex-notata (Fall) to transmit aster yellows. Am. J. Bot., 24, 316-327. 
Marwitz R., Petzold H., Özel M., 1974. Untersuchungen zur Übertragbarkeit des möglichen Erregers der Triebsucht des Apfels auf einen krautigen Wirt. Phytopath. Z., 81, 85-91.

Meignoz R., Caudwell A., Kuszala C., Schneider C., Larrue J., Fleury A., Boudon E., 1982. Serological purification and visualization in the electron microscope of the grapevine flavescence dorée (FD) pathogen (MLO) in diseased plants and infectious vectors extracts. 4th Int. Congress of IOM Tokyo, 1-7 sept. $180 \mathrm{p}$.

Nakamura S., Saito T., Yamamoto M., 1978. Studies on clover phyllody (part 2). Effects of temperature upon the appearance of symptom. J. Agric. Sc., Tokyo, 23 (2), 97-101.

Robinson J. C., Schwabe W. N., 1977. Studies on the regeneration of apple cultivars from root cuttings I : propagation aspects. $J$. Hortic. Science, 52, 205-220.
Rui D., 1950. Una malattia inedita : la virosi a scopazzi del melo. Humus, 6 (11), 7-10.

Schaper H., Seemuller E., 1982. Condition of the phloem and persistence of mycoplaslike organisms associated with apple proliferation and pear decline. Phytopathology, 72 (7), 736-742.

Schmid G., 1965. Five and more years of observations on the proliferation virus of apples in the field. Zaštita Bilja, 16, 285-291.

Seemuller E., 1976. Investigation to demonstrate mycoplasmalike organisms in diseased plants by fluorescence microscopy. Xth International Symposium on fruit tree virus diseases. Acta Horticulturae. Heidelberg, 67, 109-111.

Sinha R. C., 1979. Purification and serology of mycoplasmalike organisms from aster yellows-infected plants. Can. J. Plant Pathol., 1 (2), 65-70. 